\title{
Per il testo degli scritti filosofici di Apuleio
}

\section{Carlo M. Lucarini}

Università degli studi Palermo, Piazza Marina, 61, 90133 Palermo, Italia, carlo.lucarini@unipa.it

For citation: Carlo M. Lucarini. Per il testo degli scritti filosofici di Apuleio. Philologia Classica 2019, 14 (2), 324-331. https://doi.org/10.21638/11701/spbu20.2019.212

\begin{abstract}
The aim of this paper is to cast new light on the textual constitution of some passages of Apuleius' Opuscula philosophica. A new edition of this text has been recently published by G. Magnaldi for the Scriptorum classicorum bibliotheca Oxoniensis (2019). In the present paper I discuss about 40 passages, for the most of which I propose a new conjecture (my knowledge of the readings of the manuscripts is based only on the reports of the existent editions). For istance: De deo S. 3, 9 instead of disputatione read depravatione; De deo S. 13, 4 sed differunt ab his passione should be deleted; De Plat. 1, 3, 6 ad should be deleted; De Plat. 1, 15, 4 add ne <ocius> esculenta; De Plat. 1, 16, 1 instead of ad celeritatem facilius se movendi read ad facilitatem celerius se movendi; De Plat. 2, 12, 1 extraria should be deleted; De Plat. 2, 22, 7 read adflictatio eo quod, si; De mu. 10, 2 read est <ventus $>$ nisi; De mu. 13, 4 add vero $<$ vocant $>\dot{\alpha} \pi o$; De mu. 17, 7 read inferiores quo<s>que; De mu. 23, 3 read torrentium viscerum; De mu. 25, 1 add et <propterea> poetarum; De mu. 38, 3 instead of suadeat read praesideat.
\end{abstract}

Keywords: Apuleius, ancient philosophy, textual criticism, conjectures.

Il testo degli scritti filosofici di Apuleio è stato edito nello scorso secolo dal Thomas (1908), dal Beaujeu (1973) e dal Moreschini (1991). Nuovi importanti studi sono stati pubblicati dalla Magnaldi (2011, 2012 a, 2012 b, 2014), la quale ha appena pubblicato una nuova e importantissima edizione per la Bibliotheca Oxoniensis (Magnaldi 2019). Proporrò qui una serie di congetture, basandomi sulle edizioni e gli studi citati. ${ }^{1}$

De deo Socratis 2, 7: quorum in numero sunt illi duodecim situ nominum in duo versus ab Ennio coartati. Apuleio parla qui dei dodici dèi, i cui nomi Ennio (Ann. 240-241 S.) è riuscito a disporre in due vv. L'archetipo aveva duodecim numero situ: si è espunto numero (ms. H, W. A. Baehrens), ma situ da solo non convince. Io suggerisco numerositate nominum "grazie alla forma metrica dei nomi"; Apuleio vorrebbe cioè dire che Ennio ha potuto riunire i nomi degli dèi in due soli vv. grazie alla favorevole forma metrica di tali nomi.

De d. S. 3, 1: ceterum profana philosophiae turba imperitorum, vana sanctitudinis, priva verae rationis, inops religionis, inpos veritatis, scrupolosissimo cultu, insolentissimo spretu deos neglegit, pars in superstitione, pars in contemptu timida vel tumida. Credo abbia ragione Goldbacher a ipotizzare lacuna dopo deos, poiché è strano dire che qualcuno trascura (neglegit) gli dèi scrupolosissimo cultu e anche la distinzione fra scripolosissimus cultus / insolentissimus spretus e timidi / tumidi suggerisce che il cultus si riferisca ai primi, il neglegere ai secondi. Si potrebbe supporre che sia caduto qualcosa come $\langle$ emit vel $>$ : cfr.

${ }^{1}$ Giuseppina Magnaldi ha avuto la gentilezza di farmi leggere le bozze di stampa delled. oxoniense appena uscita (quando ho scritto questo articolo essa non era, infatti, ancora pubblicata); citerò il testo secondo tali bozze: eventuali differenze fra il testo da me trascritto e quello pubblicato nelled. sono da imputare al processo editoriale. Ho discusso molti di questi passi con i miei studenti palermitani, che ringrazio per le stimolanti discussioni, all'interno di un corso di filologia classica nell'anno accademico 2018/2019.

(C) St. Petersburg State University, 2019 
Pers. Sat. 2, 3 (prece poscis emaci), 2, 29-30 (qua tu mercede deorum / emeris auricolas) ove il contesto è del tutto analogo al nostro.

De d. S. 3, 9: sed nunc non de errorum disputatione, sed de naturae distributione disserimus. L'espressione disserere de disputatione è sorprendente e Lipsio ha proposto di correggere il sostantivo in dispunctione, che è felice paleograficamente, ma problematico per il senso e mai attestato in Apuleio ${ }^{2}$. Io suggerisco depravatione (cfr. nelle linee precedenti quanquam plerique se depravaverint); la corruzione di de-in dis-potrebbe essere stata causata da disputatione.

De d. S. 5, 7: nam et ius iurandum Iovis iurandum dicitur, ut ait Ennius. Apuleio sta cercando di mostrare la sacralità del giuramento e cita a testimone Ennio $\left(380 \mathrm{R}^{3}\right.$.: o Fides alma apta pinnis, et ius iurandum Iovis), che dice il giuramento di Giove. Forse bisogna leggere nam ius iurandum et Iovis iurandum dicitur: il senso sarebbe lo ius iurandum viene chiamato anche Iovis iurandum (il che ne dimostra la sacralità, facendone proprietà di Giove).

De d. S. 13, 3-4: quippe, ut fine conprehendam, daemones sunt genere animalia, ingenio rationabilia, animo passiva, corpore aeria, tempore aeterna. ex his quinque, quae commemoravi, tria a principio eadem quae nobis, quartum proprium, postremum commune cum diis immortalibus habent, sed differunt ab his passione. quae propterea passiva non absurde, ut arbitror, nominavi, quod sunt iisdem, quibus nos, turbationibus mentis obnoxii. È strano che Apuleio, dopo aver detto che i dèmoni hanno in comune tre caratteristiche con gli uomini, una con gli dèi e unaltra con nessuno dei due, aggiunga che i dèmoni differiscono dagli dèi passione. Dal ragionamento si dedurrebbe che tutte le quattro prime caratteristiche dei dèmoni non siano proprie degli dèi. Forse sed differunt ab his passione è una glossa aggiunta per preparare la proposizione successiva?

De d. S. 16, 4: at ubi vita edita remeandum est, eundem illum, qui nobis praeditus fuit, raptare ilico et trahere. Non credo che remeandum est da solo possa andare; forse remeandum <unde venimus $>$ est?

De Platone 1, 3, 4: quapropter inventa Parmenidae ac Zenonis studiosius exsecutus ita omnibus, quae admirationi sunt singula, suos libros explevit, ut primus tripertitam philosophiam copularet, sibique invicem necessarias partes nec pugnare inter se eas modo sed etiam mutuis adiuvare auxiliis ostenderet. Io integrerei partes $<$ esse $>$ nec $<$ non $>$ pugnare inter se (già Thomas ha integrato necessarias <esse >).

De Pl. 1, 3, 6: cum principes harum familiarum inpolitas sententias et inchoatas auditoribus tradidissent, eas hic cum ratione limando tum ad orationis augustae honestissimam speciem induendo perfectas atque etiam admirabiles fecit. Non capisco la costruzione di induendo; credo si debba espungere ad, facendo di speciem l'oggetto di questo verbo, cfr. Sen. De ben. 4, 17, 2 (non ipsis, quae inpotentissime fecit, speciem aliquam induat recti).

De Pl. 1, 9, 5-6: et superiorem quidem partem mutabilem esse ac facilem contuenti, hanc autem, quae mentis acie videtur, et penetrabili cogitatione percipitur atque concipitur, incorruptam, immutabilem, constantem eandemque et semper esse. 6 hinc et duplicem rationem interpretationemque dicit. Forse et prima di semper va espunto? Cfr. e. g. Plaut. Amph. 447 (idem sum qui semper fui). Forse prima di rationem bisogna integrare <rerum $>$ ?

2 Il sostantivo è stato ipotizzato da Helm anche in Apol. 96 al posto anche qui del tràdito disputationem, ma dispositionem (Fulvius) pare decisamente migliore. 
De Pl. 1, 11, 5-6: tertium [scil.: animantium genus] ex aqua terraque coalescere; 6 et mortale genus corporum ex eo dividi terrenum atque terrestre. Non comprendo il costrutto dividi terrenum atque terrestre; il senso è chiaro ("se divise-t-il en race vivant en terre et race vivant sur terre", Beaujeu), ma credo si debba integrare dividi <in > terrenum, cfr. De Plat. 1, 11 (in quattuor species dividuntur); De Plat. 1, 18 (in partes duas dividit); De mundo 6 (in maxima divisus est maria).

De Pl. 1, 13, 2: at enim cum tres partes animae ducat esse, ratinabilem, id est mentis optimam portionem, hanc ait capitis arcem tenere; irascentiam vero procul a ratione ad domicilium cordis deductam esse obsequique eam in loco respondere sapientiae; cupidinem atque adpetitus, postremam mentis portionem, infernas abdominis sedes tenere. Non capisco la costruzione di obsequique ... sapientiae né le traduzioni mi aiutano ("la parte collerica, invece, è stata messa in basso, lontano dalla ragione, nella sede del cuore, e da lì obbedisce e risponde alla saggezza", Dal Chiele; "l'élément irascible est abaissé loin de la raison, dans le cour, où il réside, et en cette position déférente répond aux appels de la sagesse", Beaujeu). Io credo che in loco respondere sia una glossa; per conservare questo segmento dovremmo avere qualcosa come deductam esse atque in eo loco eam respondere obsequique sapientiae.

De Pl. 1, 14, 8: quod quidem aliis animantibus ad explendam victus necessitatem inferendasque ventri copias conparatum est, sed homini promptuarium potius rectae rationis et suavissimae orationis hoc datum est, ut, quae prudentia corde conceperit, ea sensa promat oratio. La bocca, che agli altri animali serve solo per mangiare, è per l'uomo strumento per esprimersi. Non comprendo come si possa tollerare sed: se quod e hoc sono in correlazione, credo che sed vada espunto (può trattarsi di una geminazione causata dal precedente est). Thomas propone dubitativamente <in > hoc, che renderebbe il periodo certo più elegante; se si accetti tale congettura, sed potrebbe essere corretto in $i d$, espungendo est dopo datum.

De Pl. 1, 15, 4: ventrem hiris intestinorum circumplexum et nexibus impeditum esse, ne esculenta et potulenta sese penetrarent, sed ut retenta paulisper utilitatem sui ac censum

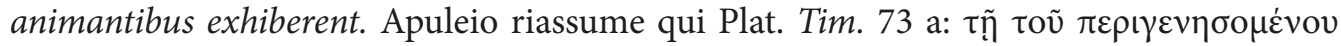

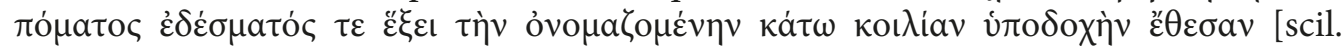

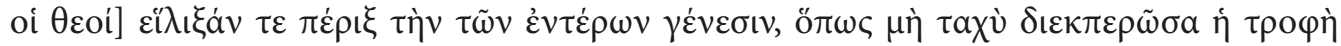

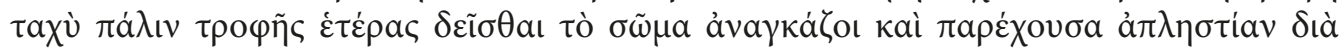

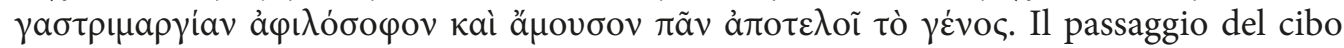
nel corpo, osservano Platone e Apuleio, non deve avvenire troppo rapidamente, perché è necessario che il corpo trattenga qualcosa del cibo. Mi pare che nel testo apuleiano sia caduto un avverbio di tempo: la frase ne esculenta et potulenta sese penetrarent significa "affinché il cibo e le bevande non pentrino", ma è evidente che Apuleio non vuole dire che le contorsioni del tubo intestinale servono affinché il cibo non penetri, ma affinché esso non pentri troppo velocente (cfr. $\tau a \chi v \dot{v}$ in Platone). Credo si debba integrare qualcosa come ne<ocius> esculenta.

De Pl. 1, 16, 1: illa etiam quae iuncturis et copulis nexa sunt, ad celeritatem facilius se movendi, haud multis sunt impedita visceribus. Forse ad facilitatem celerius se movendi?

De Pl. 1, 18, 3: eiusmodi ad aequabilitatem partibus animae temperatis, corpus nulla turbatione frangitur. alioquin invehit aegritudinem atque invalentiam et foeditatem, cum

\footnotetext{
3 Sese penetrare $=$ penetrare.
} 
inconpositae et inaequales inter se erunt. Accettando questo testo il soggetto di invehit è corpus, ma io credo che esso debbano essere iracundia et voluptas di cui si è detto immediatamente sopra, poiché invehere si dice di ciò che porta una cosa in un'altra, non di chi porta qualcosa al proprio interno, cfr. De Pl. 2, 10 (quaedam absolute bona dicimus, quae semper atque omnibus, cum absunt, invehunt commoda); ib. 2, 11 (vitia non solum turpitudinem existimationi invehere). Scriverei dunque invehunt. Il problema era già stato avvertito da W. Kroll, che aveva congetturato invehi; tale congettura sembra non sia mai stata pubblicata dallo studioso (cfr. TLL s. v. inveho 129,59 ) ed è sfuggita agli studiosi di Apuleio.

De Pl. 2, 2, 4: et illum quidem, qui natura inbutus est ad sequendum bonum, non modo sibimet intimatum putat, sed omnibus etiam hominibus, nec pari aut simili modo, verum [et] iam <suis > unumquemque acceptum esse, dehinc proximis et mox ceteris, qui familiari usu vel notitia iunguntur. Il testo è stato così costituito dalla Magnaldi ${ }^{4}$. Secondo me il pensiero è che il vir bonus è caro (acceptum) in primis a chi gli è più vicino, poi, in proporzione alla distanza, a tutti; si è quindi proposto di correggere etiam in primum liberis atque parentibus (Moreschini), ovvero di integrare prima di tale termine <parentibus et coniugi, liberis $>$ (Giusta). Una soluzione paleograficamente più economica sarebbe semplicemente correggere etiam in intimis.

De Pl. 2, 3, 2-3: honesta eadem, quae sunt bona, confidenter optare nos oportere. 3 tria genera ingeniorum ab eo sunt conprehensa, quorum praestans, egregium appellat unum, alterum deterrimum pessimumque; tertium ex utroque modice temperatum medium nuncupavit. La prima proposizione significa "e che dobbiamo scegliere con determinazione le azioni nobili, che sono anche buone" (Dal Chiele): mi pare si debba scrivere honesta, quae sunt eadem bona. Nella parte successiva l'autorevolissimo Bruxellensis (seguito dalla Magnaldi, ma non da altri editori) omette et prima di egregium. Seguendo tale testo, potremmo espungere unum e intendere "Platone chiama egregium quello che fra i tre generi di ingenium è il migliore (praestans)".

De Pl. 2, 12, 1: corporis sanitatem, vires, indolentiam ceteraque eius bona extraria, item divitias et cetera quae fortunae commoda ducimus, ea non simpliciter bona nuncupanda sunt. Credo che extraria vada espunto, poiché i beni del corpo sono sanitas, vires ecc, mentre i bona extraria sono divitiae et cetera quae fortunae commoda ducimus, cfr. De Pl. 2, 2 (corpori rebusque venientibus extrinsecus), 2, 17 (corporis et externis), 2, 23 (corporis cura et eorum, quae extrinsecus veniunt); dunque gli extraria entrano in gioco solo a partire da item.

De Pl. 2, 16, 1-2: qui sit autem pessimus, eum non solum turpem et damnosum et contemptorem deorum et inmoderatum et inhumanam atque insociabilem vitam ait vivere, sed nec cum proximis secumve congruere atque ideo non a ceteris modo verum etiam a se discrepare, nec aliis tantum sed sibi etiam inimicum esse, et idcirco hunc talem neque bonis nec omnino cuiquam nec sibi quidem amicum esse, 2 sed eum [pessimum] ${ }^{5}$ videri, quem nulla

${ }^{4}$ Che motiva la sua scelta in Magnaldi (2012 b) $490 \mathrm{sgg}$.

${ }^{5}$ La stessa Magnaldi $(2011,399$ sgg.) aveva proposto questa espunzione, ma poi nell'edizione vi ha rinunciato. A me questa espunzione pare certa: accettando il testo dei mss. bisogna supporre che con sed eum si inizi a parlare di una persona diversa rispetto a quella di cui si è parlato fino a quel momento, mentre Apuleio continua a parlare del pessimus, del quale ha cominicato a parlare all'inizio della sezione che ho trascritto. Nell'ed. la Magnaldi mette punto dopo esse. 
malignitatis superlatio possit excedere. hunc talem numquam in agendis rebus expedire se posse non solum propter inscientiam, sed quod ipse etiam sibimet sit ignotus.

Forse bisogna integrare neque bonis <neque malis $>$ nec omnino? (cfr. la fonte del

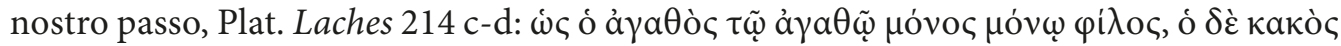

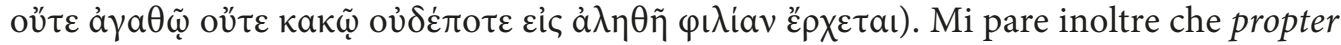
inscientiam sia troppo generico e che sia necessario integrare qualcosa che si opponga alla inscientia sui, di cui si parla immediatamente dopo. Forse propter <earum> [scil. rerum]?

De Pl. 2, 17, 2: cum igitur possessio mali ab inprudentibus capitur, usum eius et actiones oportet ab ignorantibus sustineri idcircoque peius est <nocere $>$ quam noceri, quod enim his rebus nocetur, quae sunt viliores, corporis et externis, quae vel imminui possunt vel fraudibus interire, illaesis potioribus, quae ad ipsam attinent animam. I beni che non appartengono all'anima, cioè il corpo e le cose esterne, possono essere danneggiati (noceri) dall'esterno: fraudibus interire si riferisce evidentemente alle cose che perdiamo perché qualcuno ce le sottrae, mentre imminui si riferisce ai danni fisici subiti dal corpo ${ }^{6}$. La presenza di fraudibus suggerisce l'integrazione vel <vi> inminui, cfr. Cic. De off. 1, 40; Curt. Ruf. 5, 10, 8 (quod vi non porterant, fraude adsequi temptant).

De Pl. 2, 22, 1: bonos omnes oportet inter se amicos esse, etsi sunt minus noti, et potestate ipsa, qua mores eorum sectaeque conveniunt, amici sunt habendi; paria quippe a similibus non abhorrent. Non capisco quale sia la potestas che unisce i buoni e li rende amici l'uno dell'altro. Forse honestate ipsa? Cfr. Sen. Ep. Mor. 6, 3 (multos tibi dabo qui non amico sed amicitia caruerint: hoc non potest accidere cum animos in societatem honesta cupiendi par voluntas trahit).

De Pl. 2, 22, 7: nec angetur carissimis orbatus adfectibus, vel quod ex se omnia sunt apta, quae ad beatitudinem pergunt, vel quod decreto et lege rectae rationis interdicitur eiusmodi adflictatio. 8 [et] Quod, si de tali se causa discruciet, illam aegritudinem vel propter eum, qui est emortuus, suscipiat, quasi in peiore sit parte, aut sua gratia, quod tali necessitudine doleat se privatum esse; 9 Sed neque obiti causa lamentationes suscipi oportet, si sciamus illum neque aliquid mali passum ac, si bonae fuerit voluntatis, etiam melioribus aggregatum, neque sui gratia, ut qui in se reponit omnia. Crea difficoltà et quod sia perché non si coordina a vel quod... vel quod sia perché ciò che segue sembra la spiegazione di quanto precede, poiché viene spiegato il motivo per cui decretum et lex rectae rationis interdicunt adflictationem. La difficoltà non è sfuggita all'acume della Magnaldi $(2014,66)$, che espunge et. Io scriverei adflictatio eo quod, si, cfr. De mundo 13: imparem numerum habere ventorum eo quod ortus et occasus mutentur.

De Pl. 2, 24, 3: ut omnes una mente sint invicemque sibi factum velint. Forse $<$ bene $>$ factum velint?

De Pl. 2, 24, 4: vires enim non corporis nec pecuniae collectas dominatione multorum existimandas putat cum vecordia impotentiaque, sed cum decreto communi virtutibus omnibus ornati viri incolae et omnes fundati legibus obsequuntur. Forse ornati viri et incolae omnes fundati? Per incolae omnes cfr. ps.-Caes. Bell. Afr. 74, 2, 6; Curt. Ruf. 8, 10, 6.

${ }^{6}$ Beaujeu traduce: "corps et biens extérieurs, qui peuvent être soit amoindries soit anéanties par de mauvais coups", ma in questo modo si perde la distinzione fra imminui che si riferisce al corpus e fraudibus interire, che si riferisce agli externa. 
De Pl. 2, 25, 1: moribus [et] ${ }^{7}$ huiuscemodi cunctos cives imbuendos esse dicit, ut iis in quorum tutelam et fidem res publica illa creditur auri atque argenti habendi cupido nulla sit, ne specie communi privatas opes appetant. "... non mirino a procurarsi ricchezze private, facendole sembrare di interesse pubblico" traduce la Dal Chiele. Forse bisogna integrare communis <commodi> privatas? De Pl. 2, 26 (communis commodi causam).

De Pl. 2, 27, 4: in actuosa vero civitate describit quemadmodum simul omnis hominum multitudo bonitate et iustitia conducta habeatur. Gli interpreti intendono la fine del periodo "sono invece la virtù e la giustizia a tenere unita l'intera popolazione" e questo sembra anche a me il significato, ma conduci in questo senso astratto non torva paralleli (cfr. TLL s. v. conduco 158,6$)$. Forse coniuncta?

De mundo 4, 1: haec [scil. terra] frequentatur animantibus, haec silvarum viriditate vestitur, haec fontium perennitate recreatur, haec fluminum frigidos lapsus nunc erroribus terrenis vehit, modo profundo in mari confundit. La terra in parte ospita i fiumi, in parte ne getta le acque nei mari. Credo si debba espungere in: cfr. Verg. aen. 3, 696; Curt. Ruf. 9, 4, 8 (Acesinus Hydaspi confunditur); De mundo 21 (directis obliqua confundit); TLL s. v. confundo 259, 52, ove è evidente che il costrutto con in non ha paralleli.

De mu. 10, 2: verum aliae sunt passiones, cum impulsu frigidioris aeris venti generantur. Nec enim aliud est nisi multum et vehemens in unum coacti aeris flumen. Beaujeu traduce correttamente: "mais il se produit d'autres perturbations, quand une impulsion exercée par l'air froid engendre des vents. En effet, le vent n'est autre qu'un grand et violent flux d'air rassemblé en un seul faisceau". A me non pare si possa sottintendere il soggetto di est, poiché esso in precedenza è espresso al plurale. Scriverei dunque aliud est <ventus> nisi.

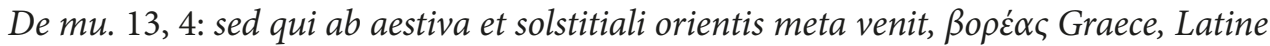

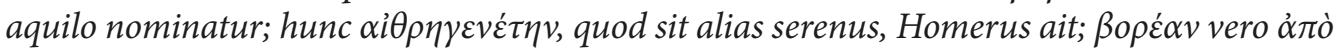
$\tau \tilde{\eta} \varsigma \beta o \tilde{\eta} \varsigma$, quod non sine clamore soleat intonare. La sintassi non mi pare corrisponda al senso richiesto; Beaujeu traduce: “... Homère le dit "aithrègénète”, parce qu’il est généralement serein; le mot "borée" dérivait de boè (= "cri”), parce que d'ordinaire il tonne en hurlant". Anch'io credo che il senso sia questo e il passo parallelo in Gellio (N. A. 2, 22, 9) con-

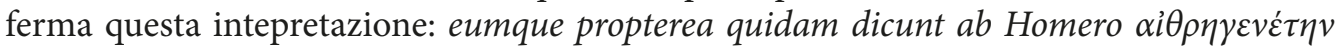
appellatum; boream putant dictum ảสò $\tau \tilde{\eta} \varsigma \beta 0 \tilde{\eta} \varsigma$, quoniam sit violenti flatus et sonori. Il testo tràdito del De mundo, tuttavia, non mi pare consentire questa interpretazione, poiché $\beta o \rho \varepsilon ́ \alpha v$... intonare non ha un verbo reggente e dovrebbe dipendere da ait, ma è evidente che l'etimologia ảंò $\tau \tilde{\eta} \varsigma \beta$ ßñ s non ha nulla a che fare con Omero. Io credo bisogni integrare qualcosa come vero <vocant $>\dot{\alpha} \pi o ́$.

De mu. 17, 7: antistites denique ipsos semiviros esse, qui audeant propius accedere, ad superna semper sua ora tollentes; adeo illis cognitum est $v i<$ rus $>$ mali, ad inferiora aeris noxii crassitate densa, inferiores quoque facilius adire atque percellere. La Magnaldi congettura cognitum est virus, Purser cognitum est vim in luogo di cognita vis est / cognitu est vis / cognitus est vis dei mss. Sono entrambe congetture ragionevoli, ma cè forse un altro problema finora sfuggito agli studiosi; io non intendo il valore di quoque: forse bisogna leggere $q u o<s>q u e$ ? Nella pericope precedente si era parlato degli animali che muoiono a causa delle esalazioni, in quella trascritta dei sacerdoti stessi e alla fine starebbe assai bene dire che le esalazioni uccidono tutti (quosque) gli esseri che tengano

7 La convincente espunzione è della Magnaldi (2012 a, 166). 
la testa vicino al terreno. Cfr. De mundo 25: denique propiores quosque de potestate eius amplius trahere.

De mu. 19, 6: aut profecto quod res est fateantur, hanc esse civilis rationis admirandam temperantiam, cum quidem de pluribus una sit facta et similis sui tota. Forse pluribus

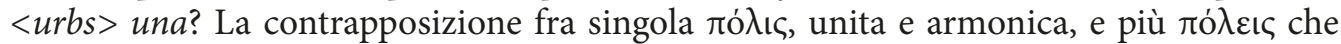
convivono all'interno di un'unica città, rendendola disomogenea e caotica, è platonica, cfr. Resp. 422 e

De mu. 23, 3: tepores frigus glaciale mitificant et brumalis austeritas terrestrium visce-

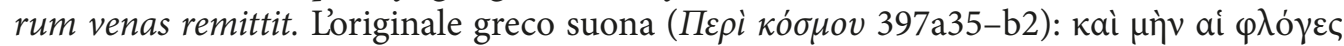

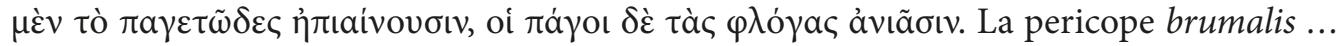
remittit non contiene alcuna allusione al calore; forse bisogna scrivere torrentium viscerum? Per torrere viscera cfr. Verg. Aen. 5, 103; Ov. Met. 8, 516; Luc. B. c. 4, 324.

De mu. 25, 1: nec ambigitur eum praestantem, sublimem sedem tenere et poetarum laudibus nomen eius consulum ac regum nuncupationibus praedicari et in arduis arcibus habere solium consecratum. Lautore afferma che sicuramente (nec ambigitur) il rector mundi è superiore a tutto, e che i poeti gli attribuiscono le caratteristiche dei consoli e dei re. Il testo dei manoscritti coordina la certezza della superiorità del rector mundi e del fatto che i poeti lo celebrano come un console o un re, ma la logica suggerisce che la lode dei poeti sia una conseguenza della superiorità del rector mundi e l'originale greco

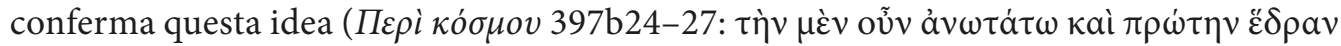

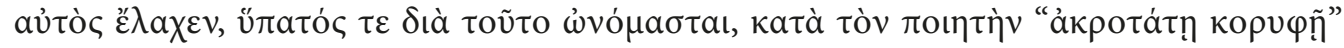

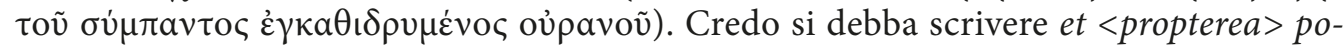
etarum.

De mu. 26, 2 : in comitatu regio armiger $<i>$ quidam, at extrinsecus singuli custodes locorum erant et ianitores et atrienses. Credo si debba leggere sungulorum custodes locorum,

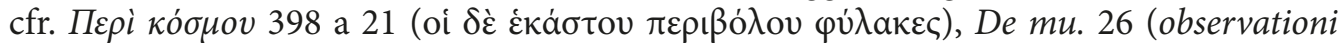
singularum rerum adpositi erant).

De mu. 28, 3: pars aquam repetens, illa inter cicures atque agrestes legibus et insitutis suis aggregabuntur, ibunt per aeris vias praepetes, quibus hoc natura largita est. Apuleio dice qui che i tre generi di animali (acquatici, terrestri e volatili) sono portati dalla loro stessa natura a abitare i luoghi loro soliti. Mentre la caratterizzazione dei pesci e degli uccelli non pone problemi, non capisco il pezzo illa ... aggregabuntur (che evidentemente si riferisce agli animali terrestri), sia perché non c’è un riferimento al fatto che essi abitano sulla terra ferma, sia perché non mi è chiaro a cosa si riferisca inter. Forse bisogna leggere illa in ter $<$ ris $>$, cicures atque agrestes, legibus...

De mu. 35, 4: et alius ad Minuciam frumentatum venit et aliis in iudiciis dies dici-

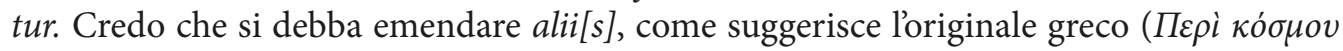

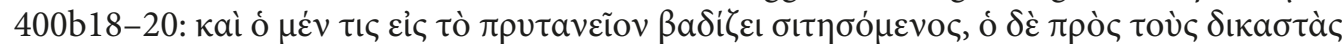
á $\pi \circ \lambda о \gamma \eta \sigma o ́ \mu \varepsilon v o \varsigma)$ e il fatto che negli altri casi si hanno singolari (alius, reus, accusator, ille, hic).

De mu. 38, 3: Clotho praesentis temporis habet curam, ut ipsis actionibus suadeat, ne cura sollers rebus omnibus desit. Atropo si occupa del passato, Lachesi del futuro, Cloto del presente. Trovo assai strano actionibus suadeat: cosa significa "persuadere le azioni"? Forse actionibus praesideat? Cfr. De mu. 35 (iudiciis praesidebunt). 


\section{Bibliografia}

Beaujeau J. (éd). Apulée, Opuscules philosophiques. Paris, Les Belles Lettres, 1973.

Dal Chiele E. Apuleio, De Platone et eius dogmate. Bologna, Bononia University Press, 2016.

Magnaldi G. Antiche note di lettura in Apul. Plat. 193, 223, 242, 248, 253, 256 e Socr. 120. RFIC 2011, 139, 394-412.

Magnaldi G. Usus di copisti ed emendatio nel De Platone di Apuleio. MD 2012, 68, 153-172 (=Magnaldi 2012a).

Magnaldi G. Antiche tracce di 'apparato' nel testo tràdito di Apuleio filosofo. Lexis 2012, 30, 478-492 (= Magnaldi 2012b).

Magnaldi G. 2014: Loci vexati nel 'De Platone' di Apuleio (190, 194, 206, 219, 229, 230, 241, 247, 252). Exemplaria classica. 2014, 18, 55-71.

Magnaldi G. Apuleius, Opera philosophica. Oxford, OUP, 2019.

Moreschini G. (ed.) Apuleius, De philosophia libri. Stuttgart - Leipzig, Teubner, 1991.

Thomas P. (ed.) Apuleius, De philosophia libri. Leipzig, Teubner, 1908.

Received: June 12, 2019

Accepted: September 17, 2019 